\title{
EPIDEMIOLOGÍA DEL CÁNCER DE ESTÓMAGO EN UN CENTRO DE REFERENCIA DEL ECUADOR.
}

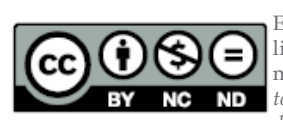

Este artículo está bajo una Escencia de Creative Con mons de tipo Reconocimienmons de tipo Reconocimien-
o- No comercial - Sin obras OPEN ACCESS derivadas 4.0 International.

1. Hospital de Especialidades Eugenio Espejo. Médico tratante del Servicio de Oncología. Quito - Ecuador. 2. Hospital de Especialidades Eugenio Espejo. Médico residente del Servicio de Oncología. Quito - Ecuador

Acuña Sonia

orcid.org/0000-0003-3893-8101

Solís Paola

orcid.org/0000-0003-1725-232X

Oñate Paola

orcid.org/0000-0002-2457-2279

Martínez Erika

orcid.org/0000-0001-5486-1672

Chaves Santiago

orcid.org/0000-0001-6242-7323

*Corresponding author: Acuña Sonia

E-mail: sonia.valeria@live.com

Article history

Received: 22 - Apr - 2020

Accepted: 11 - Ago - 2020

Publish: 1 - Sep - 2020

STROBE 2008 Check List statement: The authors have real the STROBE 2008 Check List and the manuscript was prepared and revised according to the STROBE 2008 Checklist.

Conflict of interest: All authors declared that there are no conflicts of interest.

Financial disclosure: The authors have no financial relationships relevant to this article to disclose

Authors' contribution: All the authors analyzed and interpreted the data, drafted the article and critically revised the article. All the authors reviewed and approved the final manuscript.

\section{Resumen}

\section{Introducción}

El cáncer gástrico es el tumor maligno más frecuente del Ecuador. A pesar de los avances en los métodos de diagnóstico y de las terapias oncológicas, la mayoría de los casos se diagnostican en etapas avanzadas de la enfermedad con pobre pronóstico y alta mortalidad. El objetivo de este estudio fue determinar las características epidemiológicas en pacientes atendidos con cáncer gástrico en un centro de referencia del Ecuador.

\section{Pacientes y métodos}

Fue un estudio retrospectivo transversal, fueron elegibles para participar del estudio pacientes atendidos en el Servicio de Oncología del Hospital de Especialidades Eugenio Espejo (HEEE) entre marzo del 2012 y marzo del 2017. Los datos epidemiológicos, clínicos y relacionados con el CG, se recopilaron de las historias clínicas.

\section{Resultados}

Durante el período de estudio se evaluaron 159 pacientes, la mediana de edad fue 61 años y el 52,80 \% fueron del sexo masculino. El síntoma más frecuente al diagnóstico fue el dolor abdominal en $59 \%$ de los casos. El estadio de la enfermedad en $72 \%$ de los pacientes fue loco regionalmente avanzado y diseminado. Aquellos pacientes que se sometieron a cirugía; presentaron mayor sobrevida.

\section{Conclusión}

El $50 \%$ de los casos fueron diagnosticados en etapas avanzadas de la enfermedad y se asociaron a pobre pronóstico y alta mortalidad. Las características clínicas y epidemiológicas de este primer estudio realizado en el HEEE permitirán establecer medidas que permitan mejorar el screening y diagnóstico precoz de los pacientes.

Palabras clave: Cáncer de estómago, Sobrevida libre de enfermedad, Estimación de Kaplan - Meier, Recurrencia, Quimioterapia

Forma de citar este artículo: Acuña S,

Solís P, Oñate P, Martínez E, Chaves

S. EPIDEMIOLOGÍA DEL CÁNCER

DE ESTÓMAGO EN UN CENTRO DE

REFERENCIA EN ECUADOR. Rev Med

Vozandes. 2020; 31 (2): 19 - 25 


\section{Abstract}

Keywords: Stomach Neoplasms, Disease-Free Survival , Kaplan-Meier Estimate, Recurrence, Chemotherapy

\section{EPIDEMIOLOGY OF GASTRIC CANCER IN A REFERRAL CENTER IN ECUADOR.}

\begin{abstract}
Introduction
Gastric cancer is the most common malignant tumor in Ecuador. Despite advances in diagnostic methods and cancer therapies, most cases are diagnosed in advanced stages of the disease with poor prognosis and high mortality. The objective of this study was to determine epidemiological characteristics in patients treated with gastric cancer at a reference center in Ecuador.
\end{abstract}

\begin{abstract}
Patients and methods
It was a cross-sectional retrospective study, patients were eligible in the Oncology Service of the Hospital de Especialidades Eugenio Espejo (HEEE) between March 2012 and March 2017. data were collected from medical records.
\end{abstract}

\begin{abstract}
Results
during the study period 159 patients were evaluated, the median age was 61 years and $52.80 \%$ were male. The most common symptom of diagnosis was abdominal pain in $59 \%$ of cases. The stage of the disease in $72 \%$ of patients was regionally advanced and widespread. Those patients who underwent surgery; presented more survival.

Conclusions

$50 \%$ of cases were diagnosed in advanced stages of the disease and were associated with poor prognosis and high mortality. The clinical and epidemiological characteristics of this first study carried out in the HEEE, will allow to establish measures to improve the screening and early diagnosis of patients.
\end{abstract}

\section{Introducción}

El cáncergástrico (CG) representauno de los principalesproblemas de salud a nivel mundial, los países de mayor prevalencia son los asiáticos y aunque, su incidencia ha disminuido, continúa siendo uno de los cánceres más frecuentes ${ }^{(1)}$.

En el Ecuador, el CG constituye un problema de salud pública. De acuerdo con datos de la agencia gubernamental - Instituto Nacional de Estadísticas y Censos (INEC), en 2018 se reportaron 1687 defunciones, respondiendo por el 2,3\% de la mortalidad total con 9.8 casos por 100000 habitantes ${ }^{(2,3)}$. Estos datos son diferentes de los reportados en Latinoamérica donde los cánceres de mayor mortalidad son los de pulmón y mama ${ }^{(4)}$. Existe una distribución de la incidencia y de la mortalidad asociada a las poblaciones de altura, muchas de las teorías que se han postulado están relacionadas con la concentración de minerales en el suelo o con la presión de oxígeno(5).

La carga de la enfermedad atribuible al cáncer es del 7,8\% del total de años de vida ajustados por discapacidad - disability- adjusted life-years (DALYS) a nivel mundial, el CG aporta con el 0,7\% del total de DALYs(6). . En Latinoamérica, el estudio de GBD 2017 Stomach Cancer Collaborators mostró que la tasa de DALYs corregido para la edad es $253 \cdot 8$ (234.7 a $275 \cdot 1$ ) por 100000 habitantes $^{(7)}$. Esto muestra la relevancia del CG y la necesidad de realizar un diagnóstico precoz junto con el desarrollo de programas de detección temprana de la enfermedad.

Debido al aumento en las tasas de obesidad en países desarrollados y en vías de desarrollo, la localización del CG al momento del diagnóstico ha cambiado, antes de la segunda guerra mundial la ubicación más frecuente era en la región distal del estómago, sin embargo, en las últimas décadas la mayoría de los casos se presentan en el tercio proximal (curvatura menor, cardias y unión gastroesofágica) ${ }^{(8)}$. 
Con relación a los factores de riesgo estudiados, las asociaciones más fuertes son: infección por Helicobacter Pylori, tabaquismo, alto consumo de sal y la obesidad(9).

El cáncer gástrico a menudo es diagnosticado en etapas avanzadas de la enfermedad y al momento no existe un método de pesquisa estandarizado. Estudios realizados en el Japón han demostrado que estrategias de screening están asociadas a alto costo y bajo rendimiento, por lo que no es aplicable incluso en países de la misma región (10).

El diagnóstico se lo realiza mediante biopsia tomada por endoscopia digestiva alta, su principal contribución es la determinación del subtipo histológico - intestinal o difuso, siendo este último el que presenta asociaciones genéticas más importantes y presentaciones o diagnósticos a edad más temprana (11).

Los tratamientos disponibles para esta patología dependen del estadio al momento del diagnóstico, de esta forma, en estadios precoces (enfermedad localizada), se pueden instaurar medidas con tratamiento curativo como la gastrectomía subtotal y total ${ }^{(12)}$

Respecto a las terapias sistémicas, los tratamientos quimioterápicos son considerados neoadyuvantes o adyuvantes (13) - esquemas perioperatorios, o el plan Mcdonald (tratamientos sistémicos concurrente con radioterapia adyuvante) ${ }^{(14)}$, además de tratamientos sistémicos paliativos y cuidados paliativos(15)

El objetivo de este estudio fue conocer las características de los pacientes con cáncer gástrico y compararlas con los reportes a nivel mundial para explorar los posibles determinantes de pronóstico en nuestra población.

\section{Materiales y Métodos}

\section{Selección de la Cohorte}

Este fue un estudio de cohorte retrospectivo transversal. Fueron elegibles para participar del estudio pacientes $\geq 15$ años, de ambos sexos, atendidos en el servicio de Oncología del HEEE entre marzo del 2012 y febrero del 2017, con diagnóstico histopatológico de cáncer de estómago (CIE 10: C16), en cualquier estadio.

Pacientes con dados incompletos en sus prontuarios y sin datos de seguimiento (pérdida de follow-up) fueron excluidos del análisis.

Los pacientes fueron acompañados hasta el 8 de mayo del 2018, con el objetivo de que el último paciente incluido tenga por lo mínimo un año de seguimiento.

Variables relacionadas con pronóstico y evolución de la enfermedad, como: edad, sexo, síntoma de presentación, duración de la sintomatología previo al diagnóstico, método de toma de muestra para el diagnóstico, subtipo histológico, localización, cirugía (si fue realizada y tipo), estudios de estadificación y re-estadificación, tratamientos empleados (cirugía, quimioterapia, radioterapia) y fecha de fallecimiento (cuando correspondía) se obtuvieron de las historias clínicas de los pacientes.

Para el presente manuscrito se tomaron en consideración las normativas STROBE, siguiendo a cabalidad sus lineamientos

\section{Análisis estadístico}

El análisis estadístico fue realizado con el software Epi Info versión 7.2.2. y con el software PSPP (GNU pspp 1.2.0). Datos cuantitativos fueron descritos como media y desvíos estándar. Datos categóricos fueron presentados como contajes y porcentajes. Datos categóricos fueron comparados por Chi-cuadrado o test de Fisher.

Curvas de sobrevida fueron generadas usando el teste de Kaplan Meier y comparadas con la prueba de long-rank, valores de $\mathrm{P}<0,05$ fueron considerados estadísticamente significativos.

\section{Resultados}

Caracterización de la muestra

Entre 01 de marzo de 2012 y 27 de febrero del 2017, 159 pacientes evaluados en el servicio de Oncología del HEEE cumplieron los criterios de inclusión - exclusión y participaron de este estudio. Las características clínicas de los pacientes al diagnóstico se muestran en la tabla 1.

Tabla 1. Datos Clínicos de demográficos de los pacientes al diagnóstico del $\mathrm{CH}$ en el HEEE.

\begin{tabular}{|c|c|}
\hline Variable & Resultado \\
\hline Edad (años) & $61(\min 15, \max 91)$ \\
\hline $\begin{array}{l}\text { Sexo masculino no. } \\
(\%)\end{array}$ & $85(53,5)$ \\
\hline \multicolumn{2}{|l|}{ Síntomas no. (\%) } \\
\hline Dolor abdominal & $96(59,6)$ \\
\hline Dispepsia funcional & $26(16)$ \\
\hline Nausea y vómito & $13(8)$ \\
\hline Diarrea & $3(2)$ \\
\hline $\begin{array}{l}\text { Duración de síntomas } \\
\text { (días) }\end{array}$ & $210(\min 7, \max 765)$ \\
\hline \multicolumn{2}{|l|}{ Histopatología no. (\%) } \\
\hline Difuso & $68(43)$ \\
\hline Anillo de sello + difuso & $49(31)$ \\
\hline Intestinal & $8(5)$ \\
\hline No concluyente & $26(16)$ \\
\hline No reportado & $8(5)$ \\
\hline \multicolumn{2}{|l|}{ Localización no. (\%) } \\
\hline Tercio medio & $79(49)$ \\
\hline Tercio inferior & $48(30)$ \\
\hline \multicolumn{2}{|l|}{ Tercio superior 20 (12) } \\
\hline No identificado 12 (9) & \\
\hline
\end{tabular}

Fuente: elaborado por los autore 
La endoscopia digestiva alta se realizó en 153 pacientes $(95,6 \%)$, las biopsias obtenidas se enviaron para estudio anatomopatológico, sin embargo, el estudio fue no concluyente en 26 pacientes sea por muestra insuficiente 0 falta de disponibilidad de técnicas inmuno-histoquímicas; además se pudo realizar una ubicación anatómica, misma que no fue especificada en el informe en 12 pacientes.

\section{Estadificación y tratamiento}

Mediante tomografía simple y contrastada de abdomen realizada a 139 pacientes $(87,4 \%$ ) se pudo establecer la extensión de la enfermedad, de esta forma: $55.3 \%$ de los casos (88 pacientes) presentaron enfermedad a distancia (Tabla 2).

Tabla 2. Sitio de metástasis diagnosticada por estudio tomográfico

\begin{tabular}{|lc|}
\hline Sitio de metástasis & Pacientes, $\mathbf{n}(\%)$ \\
Hepática & $30(18,6)$ \\
Pulmonar & $9(5,6)$ \\
Ganglios distantes & $29(18)$ \\
Peritoneal & $11(6,8)$ \\
Otros & $7(4,3)$ \\
\hline
\end{tabular}

Fuente: elaborado por los autores

En el gráfico 1 se evidencia el estadio tumoral presentado en los pacientes de nuestro estudio siendo el estadio clínico IV el más importante con el 37 \% (59 casos) seguido del estadio III con el $35 \%$ de los casos.

El $55.3 \%$ (86 pacientes) de los casos se sometieron a tratamiento quirúrgico, de estos, el 36\% se realizó gastrectomía parcial (58 pacientes) y el $17.6 \%$ restante (28 pacientes) gastrectomía total. El hallazgo histopatológico mostró más de 15 ganglios en 21 pacientes $(27 \%)$, y menos de 15 ganglios en 56 pacientes $(72.2 \%)$

El $59,7 \%$ de los pacientes ( $n=95$ ) recibieron tratamiento sistémico, de estos el $28.9 \%(n=46)$ se sometieron a tratamiento adyuvante, el $8.8 \%(n=14)$ recibieron terapia neoadyuvante y el $15.1 \%$ restante $(n=24)$ quimioterapia paliativa. En la tabla 3 se resumen los distintos esquemas de quimioterapia que recibieron los pacientes.

Tabla 3 Esquema de quimioterapia administrada

\begin{tabular}{|ll|}
\hline Esquema de quimioterapia & Pacientes, $\mathbf{n}(\%)$ \\
FOLFOX & $32(20)$ \\
FOLFIRI & $4(2,5)$ \\
CAPEOX & $34(21)$ \\
DCF & $3(2)$ \\
5 fluoracilo + cisplatino & $3(2)$ \\
Carboplatino + paclitaxel & $2(1)$ \\
MacDonald & $3(2)$ \\
Otros & $14(9)$ \\
Sin dato & $64(40)$ \\
FOLFOX: 5 fluoracilo, oxaliplatino y leucovorina. FOLFIRI: 5 \\
fluoracilo, irinotecan y leucovorina. CAPEOX: capecitabine y \\
Oxaliplatino. DCF: docetaxel, cisplatino y 5 fluoracilo \\
\hline
\end{tabular}




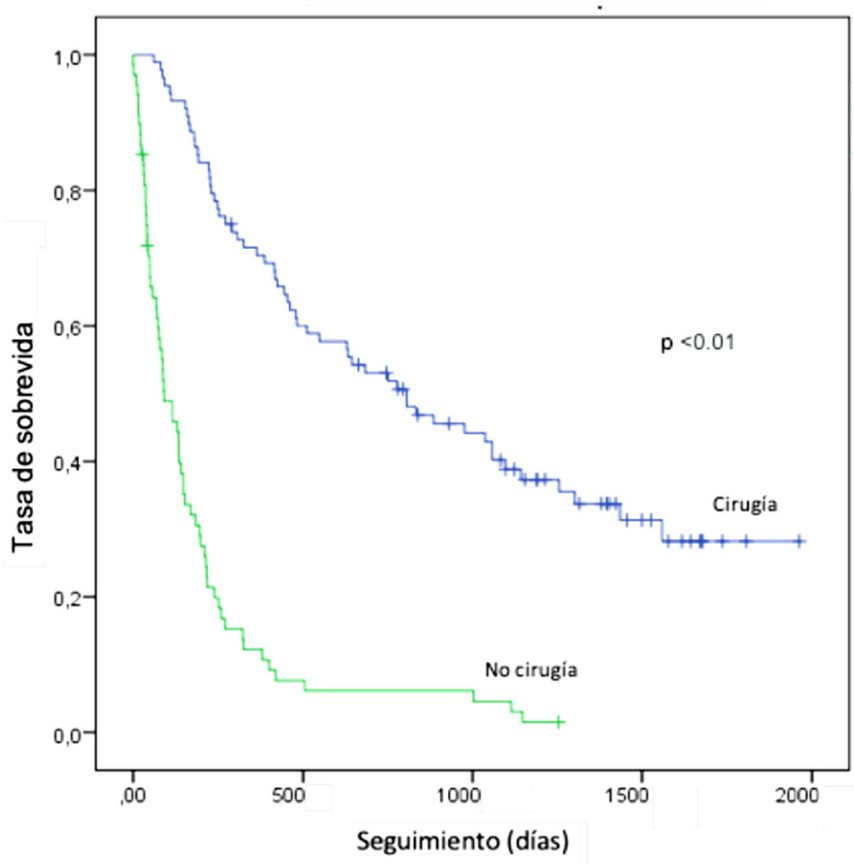

Gráfico 3. Sobrevida en pacientes que se sometieron a cirugía vs no cirugía

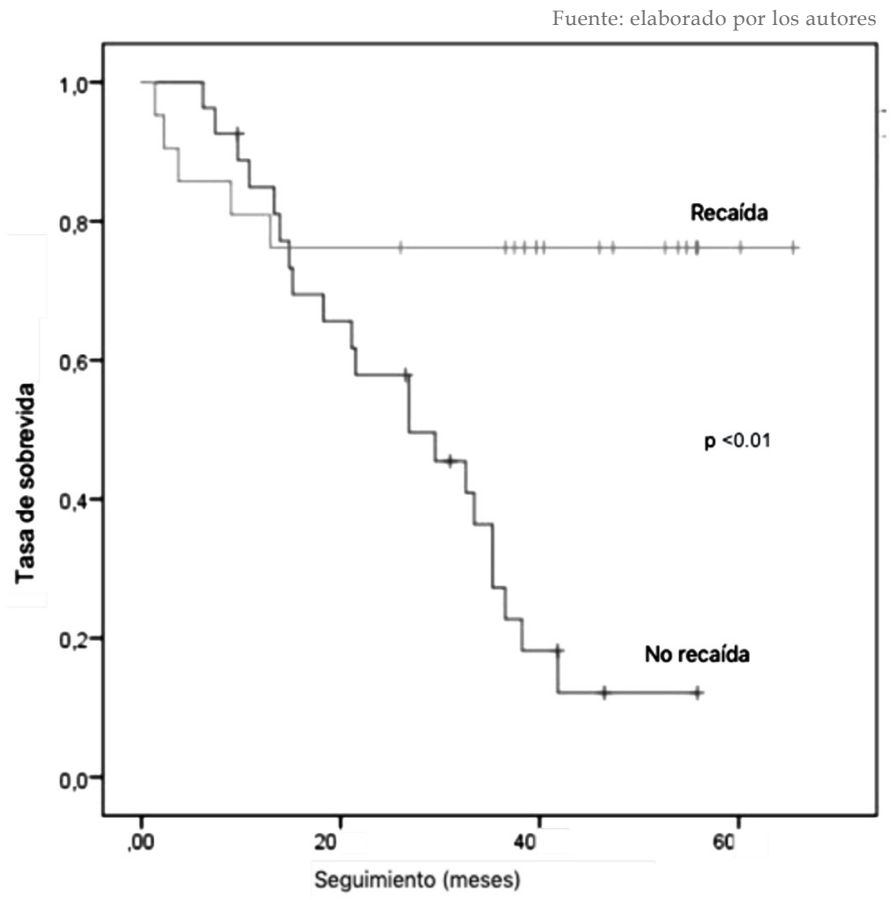

Gráfico 4. Sobrevida en pacientes que tuvieron recaída vs no recaída

Fuente: elaborado por los autores

\section{Discusión}

En nuestro estudio realizado en el Servicio de Oncología del HEEE se revisaron un total de 159 historias clínicas de pacientes que cumplieron con los criterios de inclusión, siendo 85 de los pacientes del sexo masculino, datos concordantes con un estudio realizado en el Hospital Universitario de Neiva en Colombia ${ }^{(16)}$ en el cual se encontraron 253 casos de cáncer de estómago entre el 2006 y 2008 , de los 173 pacientes, más del 50\% eran hombres. Estudio realizado en el Hospital Regional Docente de Ambato - Ecuador con una población de 57 pacientes se dedujo que dicha patología se encuentra con más frecuencia en el género masculino con $75 \%$ de casos, mientras que el género femenino alcanzo $25 \%$. El predominio del género masculino probablemente se deba a la frecuencia del hábito nocivo tabáquico, además, debemos considerar que los estrógenos son factores protectores contra el CG, tal como lo demuestra un estudio realizado en el Instituto de Tecnología de Massachusetts ${ }^{(12)}$.

En un estudio realizado en Colombia en la ciudad deBucaramangaporBermúdezy colaboradores(11) se encontró que la localización más frecuente del tumor fue antro-pilórica y la totalidad de los casos se diagnosticaron en estadio III y IV; mientras que en la población del Hospital Universitario de Neira(12) la localización más frecuente del tumor fue el antro seguido por el cuerpo gástrico, el $59,1 \%$ de los casos se diagnosticaron en estadio III y IV, a diferencia de nuestro estudio en el que encontramos que la localización más frecuente fue el cuerpo gástrico seguido del antro. Con relación al estadio al momento del diagnóstico, el $72 \%$ de los casos de nuestro estudio fueron realizados en fase III y IV, es decir el diagnóstico se lo realizó en fases avanzadas de la enfermedad, datos semejantes a los estudios realizados en Colombia, etapas asociadas a peor pronóstico. Pensamos que este retraso en el diagnóstico se debe principalmente a la falta de signos de alarma en estadios precoces de la enfermedad.

El síntoma cardinal más frecuente en nuestro estudio fue el dolor abdominal, seguido de dispepsia funcional, nausea y vómito, sintomatología parecida a la reportada en el estudio realizado por Juan Carlos Arana Reyes en 2004 en México DF (17)y en los estudios realizados en Colombia. Los autores también reportaron epigastralgia, pérdida de peso y vómito ${ }^{(12)}$ Según un estudio realizado por el servicio de gastroenterología del Hospital Clínic de Barcelona en el momento del diagnóstico, el síntoma más frecuente fue la pérdida de peso, que ocurre en el $62 \%$ de los pacientes al contrario de nuestro estudio que fue del $4 \%$, seguido del dolor abdominal (59\%).

En cuanto al resultado histopatológico del cáncer gástrico según un análisis epidemiológico realizado en el periodo 2009 al 2013 en el Hospital Carlos Luis Valverde Vega en Costa Rica, la mayoría de las histologías evidenciaron que el tipo predominante es el intestinal aunque se detectaron algunos casos de estirpe difuso (18), a diferencia de nuestro estudio cuyo resultado histopatológico predominante fue el de tipo difuso en $57.14 \%$ de los casos, lo cual llama la atención ya que probablemente se deba a una características molecular asociada a una baja regulación del $\mathrm{CDH} 1{ }^{(19)}$.

Con relación al tratamiento, según un artículo publicado en el 2017 por la Universidad de Duke en Durham Carolina del Norte, Estados Unidos (20) 
el tratamiento para el cáncer gástrico consta de quimioterapia, radioterapia y tratamiento quirúrgico, con posibilidad de combinaciones (por ejemplo, cirugía seguida de quimio y radioterapia adyuvante).

En pacientes con estadio temprano, la cirugía parecería ser la mejor opción terapéutica. En nuestro estudio el 55.3 \% (86 pacientes) de los casos se sometieron a tratamiento quirúrgico, de estos el $36 \%$ se realizó gastrectomía parcial (58 pacientes) y el 17.6\% restante (28 pacientes) gastrectomía total.

El cáncer gástrico localmente avanzado se define como irresecable inicialmente, donde la sobrevida promedio para los pacientes no tratados es de 11 meses, ${ }^{(19)}$ en estos casos el objetivo primario es la resecabilidad. En nuestro estudio 17,8 \% (17 pacientes) fueron tratados con quimioterapia con objetivo de conversión a cirugía ulterior y si bien no hubo un esquema estándar utilizado muy pocos pacientes fueron resecados (21), concordante con la literatura ya que existen numerosos estudios fase 2 con distintos esquemas de quimioterapia que demuestran la posibilidad de aumentar la resecabilidad de los tumores localmente avanzados (22,23). Las guías del "Las guías del "National Comprehensive Cancer Network (NCCN)"(24) muestran que no existe un régimen óptimo único y varias opciones de manejo son posibles aún en caso de tumores resecables donde la cirugía es solo una de las opciones para los tumores locoregionalmente avanzados. Regímenes como el FLOT (docetaxel, oxaliplatino, leucovorina y fluorouracilo) son opciones posibles, aunque los estudios disponibles hasta el momento no han demostrado ventajas en sobrevida $u$ otros resultados clínicamente relevantes ${ }^{(25)}$.

En pacientes con estadio clínico II en adelante se deberá considerar quimioterapia adyuvante $\mathrm{y} / \mathrm{o}$ perioperatoria generalmente con esquema fluorouracilo solo 0 en combinación con platino y radioterapia (26), en nuestro estudio el $53 \%$ de los pacientes recibieron quimioterapia adyuvante y el 16,14\% (26 paciente) recibieron radioterapia ulterior.

El cáncer gástrico diseminado constituye el estadio IV, en este estadio la sobrevida promedio estimada sin tratamiento es de 3 meses y los objetivos del tratamiento son: tratamiento sintomático, mejorar la calidad de vida y sobrevida. Un metaanálisis de la Red Cochrane (27) mostró que la quimioterapia mejora la sobrevida (6,7 meses adicionales) en comparación con el mejor cuidado de soporte, sin embargo, la quimioterapia combinada es superior en términos de sobrevida (en un mes adicional) en comparación con el 5-FU como agente único. En nuestro estudio el $38 \%$ de los pacientes se encontraban en estadio IV, sin embargo, solo el $28 \%$ se sometieron a tratamiento onco-específico. Un mal estado general impidió que el restante de los pacientes en este estadio pueda acceder a terapias sistémicas.
La recaída se presentó en el $48 \%$ de pacientes siendo la más frecuente a nivel hepático y ganglionar lo cual fue un factor de mal pronóstico con una alta tasa de mortalidad (28).

De acuerdo con nuestros resultados, la tasa de mortalidad fue del $80.50 \%$, resultados concordantes con los datos publicados por Montero y colaboradores ${ }^{(29)}$, quienes refieren que en el Ecuador, durante los años 2004 al 2015 el CG fue responsable por 19115 muertes, siendo 10679 en hombres y 8436 en mujeres, por lo tanto, podemos afirmar que esta enfermedad tiene una alta tasa de mortalidad ${ }^{(23)}$ en virtud del diagnóstico tardío.

Una de las principales limitaciones del estudio son las inherentes a los estudios retrospectivos realizados en un único centro, lo cual no podría ser representativo de la población, sin embargo, al ser un centro de referencia para la atención de esta patología, el hospital recibe pacientes derivados de todo el país.

Datos sobre estilos de vida, muy importantes como hábito tabáquico, consumo de alcohol, consumo de frutas y verduras no se encontraron en las historias clínicas por lo que no se las pudo evaluar. Asimismo, la toma de datos de historias clínicas, así como la variabilidad en la disponibilidad de los medios diagnósticos y terapéuticos en nuestro hospital, han limitado su correcto análisis, defectos en la calidad de los registros han impedido obtener datos importantes como los esquemas de tratamiento.

\section{Conclusiones}

Se trata del primer estudio que evalúa las características epidemiológicas y de comportamiento clínico-evolutivo del CG en HEEE - Quito Ecuador. Nuestro estudio provee el soporte con relación a las características clínicas y demográficas de los pacientes que se atienden en nuestro hospital, destacando su similitud con la literatura internacional. Aunque el subtipo histológico difiere con la literatura regional e internacional, no hubo diferencia estadísticamente significativa con la sobrevida, hallazgo que puede estar relacionado con el tamaño muestral de este subgrupo. La recaída en los pacientes representa un factor adverso asociado a pobre sobrevida.

\section{Referencias}

1. Ang TL, Fock KM. Clinical epidemiology of gastric cancer. Singapore Med J. 2014;55(12):621-628.

2. Instituto Nacional de Estadísticas y Censos. Registro Estadístico de Defunciones Generales 2018. https://www.ecuadorencifras.gob.ec (Accedido 22 abril 2020)

3. Nadia M, Solange N, Daniel S, The remarkable geographical pattern of gastric cancer mor- tality in Ecuador. Med J. 2017; 51: 92 -97

4. Forman D, Sierra MS. Cancer in Central and South America: Introduction. Cancer Epidemiol. 2016;44 Suppl 1: S3-S10.

5. Montero-Oleas N, Núñez-González S, SimancasRacines D. The remarkable geographical pattern of gastric cancer mortality in ECUador. Cancer Epidemiol. 2017; 51: 92-97.
6. Institute for Health Metrics and Evaluation. GBD compare. IHME [internet] 2016 [acceso 29 de agosto de 2020]. Disponible en: http://vizhub. healthdata.org/gbd-compare

7. GBD 2017 Stomach Cancer Collaborators. The global, regional, and national burden of stomach cancer in 195 countries, 1990-2017: a systematic analysis for the Global Burden of Disease study 2017. Lancet Gastroenterol 
Hepatol. 2020 Jan;5(1):42-54. doi: 10.1016/ S2468-1253(19)30328-0.

8. Chen $Y$, Liu L, Wang $X$, Wang J, Yan Z, Cheng $J$, et al. Body mass index and risk of gastric cancer: a meta-analysis of a population with more than ten million from 24 prospective studies. Cancer Epidemiol Biomarkers Prev 2013;22:1395-408.

9. Karimi P, Islami F, Anandasabapathy S, Freedman ND, Kamangar F. Gastric cancer: descriptive epidemiology, risk factors, screening, and prevention. Cancer Epidemiol Biomarkers Prev. 2014;23(5):700-713.

10. Tramacere I, Negri E, Pelucchi C, Bagnardi V Rota M, Scotti L, et al. A meta-analysis on alcohol drinking and gastric cancer risk. Ann Oncol. 2012 Jan;23(1):28-36. doi: 10.1093/annonc/mdrl35.

11. Cho I, Kwon IG, Guner A, Son T, Kim HI, Kang $D R$, et al. Consideration of clinicopathologic features improves patient stratification for multimodal treatment of gastric cancer. Oncotarget. 2017 Jun 22;8(45):79594-79603. doi: 10.18632/oncotarget.18607.

12. Fujiwara $Y$, Fukuda S, Tsujie $M$, Ishikawa $H$, Kitani $K$, Inove $K$, et al. Effects of age on survival and morbidity in gastric cancer patients undergoing gastrectomy. World J Gastrointest
Oncol. 2017 Jun 15:9(6):257-262. doi: 10.4251/ wjgo.v9.i6.257.

13. Zheng $Y$, Zhu $X Q$, Ren $X G$. Third-line chemotherapy in advanced gastric cancer: A systematic review and meta-analysis. Medicine (Baltimore). 2017:96(24):e6884

14. Macdonald JS, Smalley SR, Benedetti J, Hundahl SA, Estes NC, Stemmermann GN, et al. Chemoradiotherapy after surgery compared with surgery alone for adenocarcinoma of the stomach or gastroesophageal junction.
N Engl J Med. 2001 Sep 6;345(10):725-30. doi: 10.1056/NEJMoa010187.

15. Kelly CM, Janjigian YY. The genomics and therapeutics of HER2-positive gastric cancerfrom trastuzumab and beyond. J Gastrointest Oncol. 2016;7(5):750 $\square 762$.

16. González, A., Benavides, E., Santofimio, D., \& Gil, F. (2015). Cáncer gástrico: características epidemiológicas, clínicas y patológicas de los pacientes atendidos en el Hospital Universitario de Neiva entre enero del 2007 y diciembre del 2012. RFS Revista Facultad De Salud, 7(2), 23-28.

17. Arana JC, Corona A. Cáncer gástrico, Med J, 2004; 47: 204-209

18. Karen R, Randall C, Análisis epidemiológico del cáncer gástrico en el servicio de cirugia hclvv 2009-2013. Med J 2015; (615): 431 - 435

19. Hu MN, Hu SH, Zhang XW, Xiong SM, Deng $\mathrm{H}$. Overview on new progress of hereditary diffuse gastric cancer with $\mathrm{CDHl}$ variants. Tumori. 2020 Aug 18:300891620949668. doi: $10.1177 / 0300891620949668$.

20. Daphna S, Manisha P, Hope U, role of chemotheraphy and radiation therapy in the motheraphy and radiation therapy in the management of gastric adenc
Ann Surg Oncol 2016; 421-435

21. Shah MA, Janjigian YY, Stoller R, Shibata S, Kemeny $M$, Krishnamurthi $S$, et al. Randomized Multicenter Phase II Study of Modified Docetaxel, Cisplatin, and Fluorouracil (DCF) Versus DCF Plus Growth Factor Support in Patients With Metastatic Gastric Adenocarcinoma: A Study of the US Gastric Cancer Consortium. $\mathrm{J}$ Clin Oncol. 2015 Nov 20;33(33):3874-9. doi: $10.1200 / J C O .2015 .60 .7465$

22. Burtness B, Gibson M, Egleston B, Mehra R, Thomas L, Sipples R, et al. Phase II trial of docetaxel-irinotecan combination in advanced esophageal cancer. Ann Oncol. 2009 Jul;20(7):1242-8. doi: 10.1093/annonc/ man787.

23. Kim GM, Jeung HC, Rha SY, Kim HS, Jung I, $\mathrm{Nam} \mathrm{BH}$, et al. A randomized phase II trial of S-1-oxaliplatin versus capecitabine-oxaliplatin in advanced gastric cancer. Eur J Cancer. 2012 Mar;48(4):518-26. doi: 10.1016/j. ejca.2011.12.017.

24. Ajani JA, D'Amico TA, Almhanna K, Bentrem DJ, Chao J, Das P, et al. Gastric Cancer, Version 3.2016, NCCN Clinical Practice Guidelines in Oncology. J Natl Compr Canc Netw. 2016 Oct;14(10):1286-1312. doi: 10.6004/ jncen.2016.0137.

25. Kang YK, Cho $\mathrm{H}$. Perioperative FLOT: new standard for gastric cancer?. Lancet. 2019:393(10184):1914-1916.

26. Smyth EC, Verheij M, Allum W, Cunningham D, Cervantes A, Arnold D; ESMO Guidelines Committee. Gastric cancer: ESMO Clinical Practice Guidelines for diagnosis, treatment and follow-up. Ann Oncol. 2016 Sep;27(suppl 5):v38-v49. doi: 10.1093/annonc/mdw350.

27. Wagner AD, Syn NL, Moehler $M$, Grothe $W$, Yong WP, Tai BC, et al. Chemotherapy for advanced gastric cancer. Cochrane Database Syst Rev. 2017;8:CD004064.

28. Zhu Z, Gong Y, Xu H. Clinical and pathological staging of gastric cancer: Current perspectives and implications [published online ahead of print, 2020 Jun 24]. Eur J Surg Oncol. 2020:S0748-7983(20)30526-6.

29.Nadia M, Solange N, Daniel S, The remarkable geographical pattern of gastric cancer mortality in Ecuador. Med J. 2017; 51:92 -97 